\title{
Preons, Standard Model, Gravity with Torsion and Black Holes
}

\author{
Risto Raitio \\ Independent Researcher, Espoo, Finland \\ Email: risto.raitio@gmail.com
}

How to cite this paper: Raitio, R. (2017) Preons, Standard Model, Gravity with Torsion and Black Holes. Open Access Library Journal, 4: e3632.

https://doi.org/10.4236/oalib.1103632

Received: April 24, 2017

Accepted: May 13, 2017

Published: May 16, 2017

Copyright (c) 2017 by author and Open Access Library Inc.

This work is licensed under the Creative Commons Attribution International License (CC BY 4.0).

http://creativecommons.org/licenses/by/4.0/

\section{c) (†) Open Access}

\begin{abstract}
A previous spin $1 / 2$ preon model for the substructure of the standard model quarks and leptons is complemented to provide particle classification group, preon interactions and a tentative model of black holes. The goal of this study is to analyze a phenomenological theory of all interactions. A minimal amount of physical assumptions are made and only experimentally verified global and gauge groups are employed: SLq(2), the three of the standard model and the full Poincaré group. Gravity theory with torsion is introduced producing an axial-vector field coupled to preons. The mass of the axial-vector particle is estimated to be near the GUT scale. The boson can materialize above this scale and gain further mass to become a black hole at Planck mass while massless preons may form the horizon. A particle-black hole duality is proposed.
\end{abstract}

\section{Subject Areas}

Particle Physics

\section{Keywords}

Preons, Standard Model, Gravity, Torsion, Black Holes

\section{Introduction}

The purpose of this brief note is to develop further a previous spin $1 / 2$ preon model [1] in order to strengthen its global and local group theoretic structure. The model should fulfill four requirements: 1) provide a global group structure for preons, quarks and leptons, 2) introduce preon properties such that they endorse the standard model (SM) local gauge group structure $S U(3) \times S U(2) \times U(1), 3)$ provide a basis for introducing an applicable formulation of gravity into the model, and 4) introduce tentatively a corpuscular structure for black holes. Briefly, this note is a step in search for scenario of physics 
beyond the standard model. In particular, the purpose of this note is to unify all interactions into a single phenomenological theory as solidly as possible [2].

These goals are approached as follows. The preon model [1] [3] [4] [5] fermions match exactly with those of Finkelstein [6] using the global knot algebra $S L q(2)$ structure for preons, quarks and leptons. Secondly, the construction of the preon model directly suggests the gauge group structures $S U(2)$ and

$S U$ (3) for the weak and strong interactions, respectively. Thirdly, fermion fields in Einstein-Cartan [7], or Einstein-Kibble-Sciama (EKS) [8] [9] gravity have been shown by Fabbri to yield interesting results for torsion coupling to the spin of Dirac fields [10]. This interaction is expressed as a massive axial-vector field coupling to preons. It originates from translation symmetry of the full Poincaré gauge group in the action. A model for Gedanken gravity phenomenology is in this way introduceded for energy scales, say approximately

$10^{16} \mathrm{GeV} \leq E \leq 10^{19} \mathrm{GeV}$. At these energies the axial-vector boson may materialize due to preon-antipreon annihilation in stellar collisions or in similar energy density thermal environment. At $E \geq 10^{19} \mathrm{GeV}$ the axial-vector bosons are seeds for black hole formation. Near and above Planck scale the effects of torsion are taken to dominate over those of curvature, while at astronomical scales the effects of curvature are known to dominate.

The model is constructed with minimum of basic ingredients. Only two basic fermions are assumed instead of two quarks and two leptons. All interactions are taken from the experimentally tested standard model and from the EKS general relativity with torsion. Torsion in Einstein-Hilbert gravity is zero, and in the literature this is the majority opinion. However, there is no reason not to consider the full Poincaré symmetry for gravity (see last paragraph of subsection 3.1). Duality between standard model matter particles and black holes is proposed. In principle, one is calculable from the other.

The organization of this note is the following. The preon model is described in Section 2. The particle classification global group $S L q(2)$ is discussed in Appendix. Torsional interaction of preons in EKS gravity is summarized in Section 3. A tentative model for black hole structure using the torsion field is described in Section 4. Finally, conclusions are made in Section 5. The Appendix is included to make the presentation self-contained.

\section{Preons, Quarks and Leptons}

The constituents of quarks and leptons must include an odd number of spin 1/2 particles. In [1] the case of three constituents, preons, is consider. Requiring charge quantization $\{0,1 / 3,2 / 3,1\}$ and fermionic permutation antisymmetry for same charge preons, four bound states of three preons were defined. These form the first generation quarks and leptons

$$
\begin{aligned}
& u_{k}=\epsilon_{i j k} m_{i}^{+} m_{j}^{+} m^{0} \\
& \bar{d}_{k}=\epsilon_{i j k} m^{+} m_{i}^{0} m_{j}^{0} \\
& e=\epsilon_{i j k} m_{i}^{-} m_{j}^{-} m_{k}^{-} \\
& \bar{v}=\epsilon_{i j k} \bar{m}_{i}^{0} \bar{m}_{j}^{0} \bar{m}_{k}^{0}
\end{aligned}
$$


In $(2.1)$, the $\mathrm{u}$-quark charge is divided by 2 to make a preon $\mathrm{m}^{+}$while the electron charge -1 is fractioned by 3 yielding the antiparticle of $\mathrm{m}^{+1}$. The preon masses are assumed zero, or $\approx 0$.

A feature in (2.1) with two same charge preons is that the construction provides a three-valued index for quark $S U$ (3) color, as it was originally discovered [11]. The corresponding gauge bosons are in the adjoint representation. The weak $S U(2)$ left handed doublets can be read from the first two and last two lines in (2.1). The standard model gauge structure $S U(N), N=1,2$ is emergent in this sense from the present preon model. In the same way quarklepton transitions between lines $1 \leftrightarrow 3$ and $2 \leftrightarrow 4$ in (2.1) are possible. The preon and SM fermion group structure is better illuminated using the representations of the $S L q(2)$ group in Appendix.

The preon spinor $\psi$ field equation is

$$
i \gamma^{\mu} \nabla_{\mu} \psi-X W_{\sigma} \gamma^{\sigma} \pi \psi-M \psi=0
$$

where $W_{\sigma}$ is the axial-vector field of torsion, $X$ the axial-vector-preon coupling and $\pi$ is the projection operator $\pi_{H} \psi=\psi_{H}, H=L, R$. This equation will be derived in subsection 3.2. Estimates for the preon mass $m$ and the axial-vector mass $M$ are given in Section 3.

The above gauge picture is supposed to hold in the present scheme up to the energy of about $10^{16} \mathrm{GeV}$. The electroweak interaction is in the spontaneously broken symmetry phase below energies of the order of $100 \mathrm{GeV}$ and in the symmetric phase above it. The electromagnetic and weak forces take separate ways at higher energies $\left(100 \mathrm{GeV} \ll E \ll 10^{16} \mathrm{GeV}\right)$. The weak interaction restores its symmetry but melts away due to ionization of quarks and leptons into preons. The electromagnetic interaction, in turn, stays strong towards Planck scale, $M_{\mathrm{Pl}} \sim 1.22 \times 10^{19} \mathrm{GeV}$. Likewise, the quark color and leptoquark interactions suffer the same destiny as the weak force. One is left with the electromagnetic and gravitational forces only at Planck scale.

\section{Preon Interactions}

\subsection{Preliminaries}

To build a full Poincaré group gauge theory for gravity one has to consider boosts, rotations and translations: the rotations lead to curvature and the translations to torsion in spacetime. From a different point of view, curvature arises in the form of metric from energy and torsion in the form of a connection from spin. Torsion is therefore defined on microscopic scales. Torsion requires extension of the Riemann geometry to Riemann-Cartan (RC) geometry [7]. RC gravity, or Einstein-Kibble-Sciama (EKS) [8] [9] gravity can be reduced to Einstein gravity plus torsional contributions. A theory has been developed by Fabbri [10] for gravity with torsion and spinor matter fields, which yields a massive axial-

${ }^{1}$ In (1) there is a caveat since preons of the same charge only have two degrees of freedom while three are implied in (1). Therefore one more quantum number may be needed, two preons may make a scalar field, or the leptons are bound states of their chiral components as discussed in [10]. 
vector coupled to spinors. His goal is to explain most of the open problems in the standard model of particles (and cosmology) as well as to analyze the nature of spinor fields. Here I apply the axial-vector coupling of [10] to preon interactions.

The field equations of the EKS theory of gravity are

$$
\begin{gathered}
G^{\mu v}-\frac{1}{2} g^{\mu v} G-g^{\mu v} \Lambda=\frac{1}{2} k T^{\mu v} \\
Q^{\rho \mu v}=-k S^{\rho \mu \nu}
\end{gathered}
$$

where $k=8 \pi G_{N}, Q^{\rho \mu v}$ is the torsion tensor and $S^{\rho \mu v}$ is the spin density tensor, the source of torsion [10].

In general relativity, metric is used to measure distances and angles. Connections are used to define covariant derivatives. A suitable connection must be found. In general form, a covariant derivative of a vector is defined by

$$
D_{\alpha} V^{\mu}=\partial_{\alpha} V^{\mu}+V^{\rho} \Gamma_{\rho \alpha}^{\mu}
$$

The connection $\Gamma_{\rho \alpha}^{\mu}$ has three indices: $\mu$ and $\rho$ shuffle, or transform, the components of the vector $V^{\rho}$ and $\alpha$ indicates the coordinate in the partial derivative.

Metric and connection should be unrelated. This is implemented by demanding that the covariant derivative of the metric vanishes. In this case the connection is metric-compatible. Metric-compatible connections can be divided into antisymmetric part, given by the torsion tensor, and symmetric part which includes a combination of torsion tensors plus a symmetric, metric dependent connection called the Levi-Civita connection.

In a general Riemannian spacetime $\mathbf{R}$, at each point $p$ with coordinates $x^{\mu}$, there is a Minkowski tangent space $M=T_{p} \mathbf{R}$, the fiber, on which the local gauge transformation of the $T_{x^{\mu}} \mathbf{R}$ coordinates $x^{a}$ takes place

$$
x^{\prime a}=x^{a}+\epsilon^{a}\left(x^{\mu}\right)
$$

where $\epsilon^{a}$ are the transformation parameters, $\mu$ is a spacetime index and $a$ a fiber frame index.

The dynamics of the theory is based on vierbeins (tetrads) $e_{\mu}^{a}$, not on the metric tensor $g_{\mu v}$. The Cartan connection has a primary role and it is

$$
\Gamma_{\mu \lambda v}=e_{\mu}^{a} \partial_{\lambda} e_{a v}
$$

The tensor associated with this connection is torsion tensor

$$
T_{\lambda v}^{\mu}=e_{a}^{\mu}\left(\partial_{\lambda} e_{v}^{a}-\partial_{v} e_{\lambda}^{a}\right)
$$

In [10] it is shown that the connection

$$
\Lambda_{\alpha \beta}^{\rho}=\frac{1}{2} g^{\rho \mu}\left(\partial_{\beta} g_{\alpha \mu}+\partial_{\alpha} g_{\mu \beta}-\partial_{\mu} g_{\alpha \beta}\right)
$$

is symmetric and written entirely in terms of the partial derivatives of the metric tensor, and it is called metric connection, while the torsion tensor with all lower indices is taken to be completely antisymmetric and therefore it is possible to 
write it according to the following form

$$
Q_{\alpha \sigma v}=\frac{1}{6} W^{\mu} \epsilon_{\mu \alpha \sigma v}
$$

in terms of the $W^{\mu}$ pseudo-vector, therefore called torsion pseudo-vector.

Unfortunate for the development of gravitation theory, spin was not discovered in the laboratory before 1916. Spinors were introduced in mathematics by Cartan in the 1920's and spinor wave equation was found by Dirac in 1928.

\subsection{Torsion as Axial-Vector Massive Field}

The system of field equations can be derived by the variational method from a dynamical action, whose Lagrangian function is [10]

$$
\begin{aligned}
\mathscr{L}= & -\frac{1}{4}(\partial W)^{2}+\frac{1}{2} M^{2} W^{2}-\frac{1}{k} R-\frac{2}{k} \Lambda-\frac{1}{4} F^{2} \\
& +i \bar{\psi} \gamma^{\mu} \nabla_{\mu} \psi-X \bar{\psi} \gamma^{\mu} \pi \psi W_{\mu}-m \bar{\psi} \psi
\end{aligned}
$$

where $M$ is the axial-vector mass, $X$ the preon-axial-vector coupling and $\psi$ the preon wave function. $F$ is the electromagnetic tensor

$$
F_{\alpha \beta}=\partial_{\alpha} A_{\beta}-\partial_{\beta} A_{\alpha}
$$

Let us start from the metric connection

$$
\Lambda_{\alpha \beta}^{\rho}=\frac{1}{2} g^{\rho \mu}\left(\partial_{\beta} g_{\alpha \mu}+\partial_{\alpha} g_{\mu \beta}-\partial_{\mu} g_{\alpha \beta}\right)
$$

The torsion tensor is completely antisymmetric only if some restrictions are imposed, called the metric-hypercompatibility conditions [15] [16] [17] [18] [19]. Then it can be written in the form

$$
Q_{\alpha \sigma v}=\frac{1}{6} W^{\mu} \varepsilon_{\mu \alpha \sigma v}
$$

where $W^{\mu}$ is torsion pseudo-vector, obtained from the torsion tensor after a Hodge dual. With the metric connection and the torsion pseudo-vector the most general connection can be written as a sum of $\Lambda_{\alpha \beta}^{\rho}$ and $Q_{\alpha \sigma v}$ as follows

$$
\Gamma_{\alpha \beta}^{\rho}=\frac{1}{2} g^{\rho \mu}\left[\left(\partial_{\beta} g_{\alpha \mu}+\partial_{\alpha} g_{\mu \beta}-\partial_{\mu} g_{\alpha \beta}\right)+\frac{1}{6} W^{v} \varepsilon_{\nu \mu \alpha \beta}\right]
$$

Functions $\Omega_{b \mu}^{a}$ that transform under a general coordinate transformation like a lower Greek index vector and under a Lorentz transformation as

$$
\Omega_{b^{\prime} v}^{a^{\prime}}=\Lambda_{a}^{a^{\prime}}\left[\Omega_{b v}^{a}-\left(\Lambda^{-1}\right)_{k}^{a}\left(\partial_{v} \Lambda\right)_{b}^{k}\right]\left(\Lambda^{-1}\right)_{b^{\prime}}^{b}
$$

are called a spin connection. The torsion in coordinate formalism is defined as follows

$$
Q_{\mu \nu}^{a}=-\left(\partial_{\mu} e_{v}^{a}-\partial_{\nu} e_{\mu}^{a}+e_{v}^{b} \Omega_{b \mu}^{a}-e_{\mu}^{b} \Omega_{b v}^{a}\right)
$$

and the spin connection is given by

$$
\Omega_{b \mu}^{a}=e_{b}^{v} e_{\rho}^{a}\left(\Gamma_{v \mu}^{\rho}-e_{k}^{\rho} \partial_{\mu} e_{v}^{k}\right)
$$

which is antisymmetric in the two Lorentz indices after both of them are 
brought in the same upper or lower position.

The most general spinorial connection is

$$
\boldsymbol{\Omega}_{\mu}=\frac{1}{2} \Omega_{a b \mu} \boldsymbol{\sigma}^{a b}+i q A_{\mu} \mathbb{I}
$$

where $A_{\mu}$ is the gauge potential. The spinorial curvature is using the spinorial connection

$$
\boldsymbol{F}_{\alpha \beta}=\partial_{\alpha} \boldsymbol{\Omega}_{\beta}-\partial_{\beta} \boldsymbol{\Omega}_{\alpha}+\left[\boldsymbol{\Omega}_{\alpha}, \boldsymbol{\Omega}_{\beta}\right]
$$

Let us define the decomposition of the spinor field in its left and right parts

$$
\begin{array}{ll}
\pi_{L} \psi=\psi_{L} & \bar{\psi} \pi_{R}=\bar{\psi}_{L} \\
\pi_{R} \psi=\psi_{R} & \bar{\psi} \pi_{L}=\bar{\psi}_{R}
\end{array}
$$

so that

$$
\bar{\psi}_{L}+\bar{\psi}_{R}=\bar{\psi} \quad \psi_{L}+\psi_{R}=\psi
$$

The linearly independent bi-linear spinorial quantities are the following

$$
\begin{gathered}
2 \bar{\psi} \sigma^{a b} \pi \psi=\Sigma^{a b} \\
2 i \bar{\psi} \sigma^{a b} \psi=S^{a b} \\
\bar{\psi} \gamma^{a} \pi \psi=V^{a} \\
\bar{\psi} \gamma^{a} \psi=U^{a} \\
i \bar{\psi} \pi \psi=\Theta \\
\bar{\psi} \psi=\Phi
\end{gathered}
$$

To have the most general connection decomposed into the simplest symmetric connection plus torsion terms we substitute (3.13) in (3.16) and this in (3.17). The field equations reduce to the following

$$
\nabla_{\rho}(\partial W)^{\rho \mu}+M^{2} W^{\mu}=X \bar{\psi} \gamma^{\mu} \pi \psi
$$

for torsion axial-vector and

$$
\begin{aligned}
& R^{\rho \sigma}-\frac{1}{2} R g^{\rho \sigma}-\Lambda g^{\rho \sigma} \\
& =\frac{k}{2}\left[\frac{1}{4} F^{2} g^{\rho \sigma}-F^{\rho \alpha} F_{\alpha}^{\sigma}+\frac{1}{4}(\partial W)^{2} g^{\rho \sigma}-(\partial W)^{\sigma \alpha}(\partial W)_{\alpha}^{\rho}\right. \\
& +M^{2}\left(W^{\rho} W^{\sigma}-\frac{1}{2} W^{2} g^{\rho \sigma}\right)+\frac{i}{4}\left(\bar{\psi} \gamma^{\rho} \nabla^{\sigma} \psi-\nabla^{\sigma} \bar{\psi} \rho^{\rho} \psi\right. \\
& \left.\left.+\bar{\psi} \gamma^{\sigma} \nabla^{\rho} \psi-\nabla^{\rho} \bar{\psi} \gamma^{\sigma} \psi\right)-\frac{1}{2} X\left(W^{\sigma} \bar{\psi} \gamma^{\rho} \pi \psi+W^{\rho} \bar{\psi} \gamma^{\sigma} \pi \psi\right)\right]
\end{aligned}
$$

for the torsion-spin and curvature-energy coupling, and

$$
\nabla_{\sigma} F^{\sigma \mu}=q \bar{\psi} \gamma^{\mu} \psi
$$

for the gauge-current coupling; and finally

$$
i \gamma^{\mu} \nabla_{\mu} \psi-X W_{\sigma} \gamma^{\sigma} \pi \psi-m \psi=0
$$

for the spinor field equations.

From (3.28) one sees that torsion behaves like a massive axial-vector field 
satisfying Proca field equations. Torsion has the important property that it can be separated from gauge and metric factors. Torsion and gravitation seem to have the same coupling constant. However, in [10] it is shown that using the Einstein-Kibble-Sciama field equations these two independent fields with independent sources can have independent coupling constants. It is remarkable that the equations for torsion (3.28) and curvature (3.29) are so different.

The preons interact by coupling to the axial-vector boson $W$ arising in Einstein-Kibble-Sciama theory of gravity. The interaction includes two free parameters, the coupling constant $\mathrm{X}$ and the mass $\mathrm{M}$ of the axial-vector. The coupling $X$ must be larger than the electromagnetic coupling $\alpha$ to keep the charged preons bound. The preon-preon interaction is attractive [10] providing the binding for three preon states. The mass of the axial-vector boson is estimated to be of the order of the grand unified theory (GUT) scale $10^{16} \mathrm{GeV}$. This makes the torsion interaction range very short. At all scales the $W$ couples to preons relatively strongly but to the standard model particles always weakly.

Couplings in GUT theory are of the order 0.02 at the GUT scale. With a Yukawa potential in the Schrödinger equation $V(r)=-V_{0} \exp (-a r) / r$ [20], or in our notation $-X \exp (r / M) / r$ with the physicality condition

$n+l+1 \leq \sqrt{X m M}$, one may estimate that large $M$ correlates with small preon mass $m \ll m_{\text {proton }}$. These matters deserve naturally quantitative attention.

\section{Black Hole Toy Model}

Black holes are at present under intensive study. I wish to finish with a few words of a speculative black hole scheme. The axial-vector field should appear as a physical particle whenever its production is energetically possible. At Planck scale energy the axial-vector boson serves as a seed for black hole formation causing a black hole to appear. With the growing black hole mass the fermion spins average out towards zero and torsion vanishes but the physical boson remains.

The horizon is a shell of massless preon-antipreon pairs. The number of pairs is correlated with the mass of the black hole, and they may form Cooper pairs. A prototype for the lightest black hole is a preon-antipreon- $W$ excited bound state. It is a physical state which couples to quark-antiquark and lepton-antilepton pairs. This state was called gravon in [12].

One may think of a particle-black hole duality in this model. On the particle side the fermions-i.e. preons, quarks and leptons-dominate and the axial-vector is hidden with graviton being weakly coupled. On the black hole side the axialvector is the physical particle and the preons are "hidden" forming the horizon. In principle, one is calculable from the other.

\section{Conclusions}

The preon model with spin $1 / 2$, charge 0 and $1 / 3$ and mass $m \approx 0$ constituents discussed above has a sound group theoretical basis. Both the preons and the quarks and leptons belong to two lowest representations of the global SLq(2) 
group, shown in Table A1 and Table A2 of the Appendix. With two preons and their antiparticles the standard model local gauge groups

$S U(3) \times S U(2) \times U(1)$ become visible. Preons, as Dirac spinors, are the fundamental building blocks of matter which interact above GUT scale with gravity predominantly by axial-vector boson coupling. Above the Planck energy the formation of black holes becomes possible with the axial-vector boson forming a seed for it and the chiral phase preon-antipreon pairs form the horizon. The preons coupled to the axial-vector may make the singularity of the hole softer or fade away. All the basic equations, the standard model and the torsion field Equation (3.28), are relativistic quantum equations.

Gravity and electromagnetism are the "original" long range interactions in the big bang of cyclic cosmology. The axial-vector particle is expected to have a large mass, $M \sim 10^{16} \mathrm{GeV}$. At accelerator energies axial-vector couplings to standard model particles are very small. The strong and weak forces have a short interaction range within nuclei making atoms, molecules and chemistry possible. The role of curvature needs to be quantitatively evaluated. It is assumed here to be a small correction in this torsional model near the Planck scale.

In summary, the present model is by construction identical to SM below GUT scale. Between GUT and Planck scales this model and the SM are not expected to differ much numerically because of asymptotic freedom of QCD. Above Planck scale the present black hole scenario is a first step, still subject to mathematical definition, and consistency tests. More work is needed to clarify the issues and gain consensus in gravitation with torsion above GUT scale.

\section{Acknowledgements}

I thank Luca Fabbri for useful correspondence.

\section{References}

[1] Raitio, R. (1980) A Model of Lepton and Quark Structure. Physica Scripta, 22, 197. https://doi.org/10.1088/0031-8949/22/3/002

[2] Coleman, S. and Mandula, J. (1967) All Possible Symmetries of the S Matrix. Physical Review, 159, 1251-1256.

[3] Raitio, R. (2016) Combinatorial Preon Model for Matter and Unification. Open Access Library Journal, 3, e3032.

[4] Raitio, R. (2017) On the Conformal Unity between Quantum Particles and General Relativity. Open Access Library Journal, 4, e3342. https://doi.org/10.4236/oalib.1103342

[5] Raitio, R. (2017) Preons, Standard Model and Gravity with Torsion. http://vixra.org/pdf/1703.0247v1.pdf

[6] Finkelstein, R. (2016) On the SLq(2) Extension of the Standard Model and the Measure of Charge. International Journal of Modern Physics A, 32, Article ID: 1730001. https://doi.org/10.1142/S0217751X17300010

[7] Cartan, E. (1980) Sur une généralisation de la notion de courbure de Riemann et les espaces à torsion. In: Bergmann, P.G. and De Sabbata, V., Eds., Cosmology and Gravitation: Spin, Torsion, Rotation, and Supergravity, 489-491.

[8] Kibble, T. (1961) Lorentz Invariance and the Gravitational Field. Journal of Ma- 
thematical Physics, 2, 212. https://doi.org/10.1063/1.1703702

[9] Sciama, D. (1962) In Recent Developments in General Relativity (Oxford).

[10] Fabbri, L. (2017) Foundations Quadrilogy.

[11] Greenberg, O. (2009) The Color Charge Degree of Freedom in Particle Physics. In: Greenberger, D., Hentschel, K. and Weinert, F., Eds., Compendium of Quantum Physics, Springer-Verlag, Berlin Heidelberg, 109-111.

https://doi.org/10.1007/978-3-540-70626-7_32

[12] Raitio, R. (2015) The Decay of a Black Hole in a GUT Model. Open Access Library Journal, 2, e2031. https://doi.org/10.4236/oalib.1102031

[13] Thomson, W. (1868) VI.-On Vortex Motion. Transactions of the Royal Society of Edinburgh, 25, 217-260. https://doi.org/10.1017/S0080456800028179

[14] Faddeev, L. and Niemi, A. (1997) Stable Knot-Like Structures in Classical Field Theory. Nature, 387, 58-61. https://doi.org/10.1038/387058a0

[15] Fabbri, L. (2007) On a Completely Antisymmetric Cartan Torsion Tensor. Annales de la Fondation Louis de Broglie, 32, 215.

[16] Hayashi, K. (1976) Restrictions on Gauge Theory of Gravitation. Physics Letters B, 65, 437.

[17] Xin, Y. (1989) The $\Omega$-Field Theory of Gravitation and Cosmology. Astrophysical and Space Science, 154, 321-331. https://doi.org/10.1007/BF00642814

[18] Audretsch, J. and Lmmerzahl, C. (1988) Constructive Axiomatic Approach to Space Time Torsion. Classical and Quantum Gravity, 5, 1285-1295. https://doi.org/10.1088/0264-9381/5/10/008

[19] Macias, A. and Lmmerzahl, C. (1993) On the Dimensionality of Space-Time. Journal of Mathematical Physics, 34, 4540-4553. https://doi.org/10.1063/1.530355

[20] Hamzavi1, M., Movahedi, M., Thylwe, K.-E. and Rajabi, A. (2012) Approximate Analytical Solution of the Yukawa Potential with Arbitrary Angular Momenta. Chinese Physics Letters, 29, Article ID: 080302. 


\section{Appendix}

\section{Knot Theory Representations}

Early work on knots in physics goes back in time to 19th and 20th century [13] [14]. On the 21st century Finkelstein has proposed a model based on the group $S L q(2)$ [6]. This group actualizes the needs of the model of the previous Section 2.

Let us consider the simple case of two dimensional representation of the group $S L q(2)$ which is defined by the matrix

$$
T=D_{m m^{\prime}}^{1 / 2}=\left(\begin{array}{ll}
a & b \\
c & d
\end{array}\right)
$$

where $(a, b, c, d)$ satisfy the knot algebra

$$
\begin{array}{llll}
a b=q b a & b d=q d b & a d-q b c=1 & b c=c b \\
a c=q c a & c d=q d c & d a-q_{1} c b=1 & q_{1} \equiv q^{-1}
\end{array}
$$

where $q$ is defined as follows from the matrix $\epsilon$

$$
\epsilon=\left(\begin{array}{cc}
0 & \alpha_{2} \\
-\alpha_{1} & 0
\end{array}\right)
$$

The matrix $\epsilon$ is invariant under the transformation

$$
T \epsilon T^{t}=T^{t} \epsilon T=\epsilon
$$

where $T^{t}$ is $T$ transposed and $q=\alpha_{1} / \alpha_{2}$.

Higher representations of $S L q(2)$ are obtained by transforming the $(2 j+1)$ monomials

$$
\Psi_{m}^{j}=N_{m}^{j} x_{1}^{n_{+}} x_{2}^{n_{-}},-j \leq m \leq j
$$

by

$$
\begin{aligned}
& x_{1}^{\prime}=a x_{1}+b x_{2} \\
& x_{2}^{\prime}=c x_{1}+d x_{2}
\end{aligned}
$$

where $(a, b, c, d)$ satisfy the knot algebra (A.2) but $x_{1}$ and $x_{2}$ commute and $n_{ \pm}=j \pm m$, and

$$
N_{m}^{j}=\left[\left\langle n_{+}\right\rangle_{q_{1}} !\left\langle n_{-}\right\rangle_{q_{1}} !\right]^{-1 / 2}
$$

and $\langle n\rangle_{q}=\frac{q^{n-1}}{q-1}$. It is found that

$$
\left(\Psi_{m}^{j}\right)^{\prime}=\sum D_{m m^{\prime}}^{j} \Psi_{m^{\prime}}^{j}
$$

where

$$
D_{m m^{\prime}}^{j}(q \mid a, b, c, d)=\sum_{\substack{\delta\left(n_{a}+n_{b}, n_{+}\right) \\ \delta\left(n_{c}+n_{d}, n_{-}\right)}} A_{m m^{\prime}}^{j}\left(q, n_{a}, n_{c}\right) \delta\left(n_{a}+n_{b}, n_{+}^{\prime}\right) a^{n_{a}} b^{n_{b}} c^{n_{c}} d^{n_{d}}
$$

where $n_{ \pm}^{\prime}=j \pm m^{\prime}, \quad D_{m m^{\prime}}^{j}$ is a $2 j+1$ dimensional representation of the $S L q(2)$ algebra and the $A_{m m^{\prime}}^{j}$ is

$$
A_{m m^{\prime}}^{j}\left(q, n_{a}, n_{c}\right)=\left[\frac{\left\langle n_{+}^{\prime}\right\rangle_{1}\left\langle n_{-}^{\prime}\right\rangle_{1}}{\left\langle n_{+}\right\rangle_{1}\left\langle n_{-}\right\rangle_{1}}\right]^{1 / 2} \frac{\left\langle n_{+}\right\rangle_{1} !}{\left\langle n_{a}\right\rangle_{1} !\left\langle n_{b}\right\rangle_{1} !} \frac{\left\langle n_{-}\right\rangle_{1} !}{\left\langle n_{c}\right\rangle_{1} !\left\langle n_{d}\right\rangle_{1} !}
$$


Table A1. The $D^{1 / 2}$ representation of the four preons.

\begin{tabular}{ccc}
\hline$m$ & $m^{\prime}$ & preon \\
\hline $1 / 2$ & $1 / 2$ & $\mathrm{a}$ \\
$1 / 2$ & $-1 / 2$ & $\mathrm{~b}$ \\
$-1 / 2$ & $1 / 2$ & $\mathrm{c}$ \\
$-1 / 2$ & $-1 / 2$ & $\mathrm{~d}$ \\
\hline
\end{tabular}

Table A2. The $D^{3 / 2}$ representation of the standard model particles.

\begin{tabular}{cccc}
\hline$m$ & $m$ & particle & preons \\
\hline $3 / 2$ & $3 / 2$ & electron & aaa \\
$3 / 2$ & $3 / 2$ & neutrino & $\mathrm{ccc}$ \\
$3 / 2$ & $-1 / 2$ & d-quark & abb \\
$-3 / 2$ & $-1 / 2$ & u-quark & cdd \\
\hline
\end{tabular}

The oriented 2-dimensional projection of a 3-dimensional knot can be assigned three coordinates $(N, w, r)$ where $N$ is the number of crossings, $w$ is the writhe and $r$ the rotation. One can transform to new coordinates $\left(j, m, m^{\prime}\right)$. These indices label the irreducible representations of $D_{m m^{\prime}}^{j}$ of the symmetry algebra of the knot, $S L q(2)$ by setting

$$
j=N / 2, m=w / 2, m^{\prime}=(r+o) / 2
$$

This linear transformations makes half-integer representations possible. The knot constraints require $w$ and $r$ to be of opposite parity, therefore $O$ is an odd integer. The knot $(N, w, r)$ may be labeled by $D_{w / 2,(r+o) / 2}^{N / 2}(a, b, c, d)$.

One assigns physical meaning to the $D_{m m^{\prime}}^{j}$ in (A.10) by interpreting the a, b, $c$, and $d$ as creation operators for spin $1 / 2$ preons. These are the four elements of the fundamental $j=1 / 2$ representation $D_{m m^{\prime}}^{1 / 2}$ as indicated in Table A1.

For notational clarity, I use in Table A1 and Table A2. the preon names of [6]. The preon dictionary from the notation of [1] is the following:

$$
\begin{aligned}
m^{+} & \mapsto a, m^{0} \mapsto c \\
m^{-} & \mapsto d, \bar{m}^{0} \mapsto b
\end{aligned}
$$

The standard model particles are the following elements of the $D_{m m^{\prime}}^{3 / 2}$ representation as indicated in Table A2.

All details of the SLq(2) extended standard model are discussed in the review article [6], including the gauge and Higgs bosons and a candidate for dark matter. I do not, however, see much advantage for introducing composite gauge bosons in the model. Introduction of color from preons is done slightly differently in [6]. In the early universe developments there is similarity between the knot model and the present preon model. Therefore, apart from the differences in color interpretation, the model of [1] and the knot algebra of [6] are equivalent in the fermion sector.

In summary, knots having odd number of crossings are fermions and knots with even number of crossings are correspondingly bosons. The leptons and 
quarks are the simplest quantum knots, the quantum trefoils with three crossings and $j=3 / 2$. At each crossing there is a preon. The free preons are twisted loops with one crossing and $j=1 / 2$. The $j=0$ states are simple loops with zero crossings.

Submit or recommend next manuscript to OALib Journal and we will provide best service for you:

- Publication frequency: Monthly

- 9 subject areas of science, technology and medicine

- Fair and rigorous peer-review system

- Fast publication process

- Article promotion in various social networking sites (LinkedIn, Facebook, Twitter, etc.)

- Maximum dissemination of your research work

Submit Your Paper Online: Click Here to Submit

Or Contact service@oalib.com 